\title{
Prophylaxis of Macular Edema with Peroperative Intravitreal Bevacizumab in Patients with Diabetic Retinopathy Undergoing Phacoemulsification
}

Sidra Jabeen, Rizwan Khan, Ali Raza

Pak J Ophthalmol 2019, Vol. 35, No. 1

See end of article for authors affiliations

Correspondence to: Dr. Sidra Jabeen FCPS, Ophthalmology Department, Holy Family Hospital, Rawalpindi Email: sidrajabeen16@gmail.com
Purpose: To determine frequency of post-operative macular edema in patients with diabetic retinopathy receiving peroperative Intravitreal bevacizumab, as compared to controls after phacoemulsification cataract surgery.

Study Design: Randomized control trial.

Place and Duration of Study: Ophthalmology department holy family hospital Rawalpindi from July, 2016 to January, 2017.

Materials and Methods: 60 patients who reported at the health care facility with diabetic retinopathy were included in the study. Out of these 30 were kept as control and 30 as cases. Data was collected and entered into SPSS version 19.0. Numerical variables were accounted for as mean and standard deviation whereas qualitative variables were accounted for as frequency and percentages. To compare the occurrence of macular edema at completion of 6 weeks in both study groups, Pearson's chi-square test at $5 \%$ level of significance was applied. A p- value of 0.05 was considered as statistically significant. To control effect modifier by gender, and age, stratified analysis was also executed.

Results: The participants of the study had mean age of $61.97 \pm 5.7$ years. Out of the 60 patients $33(55 \%)$ were female. Analysis of both groups showed that macular edema was found in $4(13.33 \%)$ patients in control group and 17 $(56.67 \%)$ patients in group B. Most significant macular edema was seen in 11 patients in age group of 61-70 years. Gender wise stratification showed that 9 (56.5\%) female patients reported with macular edema.

Conclusion: Peroperative intravitreal bevacizumab is effective in prophylaxis of macular edema in patients with mild to moderate NPDR, as compared to controls,

Keywords: NPDR, Intravitreal bevacizumab, Diabetic Retinopathy, Macular edema, Phacemulsification surgery.
$\mathrm{M}$ acular edema (ME) is defined as abnormal thickening of the macula due to excessive accumulation of fluid in extracellular spaces of central retina. ${ }^{1}$ It is a leading cause of irreversible vision loss, in the ocular conditions such as diabetic retinopathy, venous occlusion, uveitis, after cataract surgery, ocular inflammations, and branch retinal vein occlusion ${ }^{2}$. Diabetic macular edema (DME) is a noteworthy reason for loss of central vision in diabetic patients. Post cataract surgery visual outcomes can be negatively affected by diabetic maculopathy ${ }^{3}$. 
It has been proposed that among diabetic patients undergoing uncomplicated cataract surgery 22\% develop an increment of $>30 \%$ in central macular thickness as quantified by optical coherence tomography $(\mathrm{OCT})^{3}$. Another study showed an increment of maximum macular thickness of at least $11 \%$ was found in $25.7 \%$ of the diabetic retinopathy (DR) eyes undergoing cataract surgery, but no such increase occurred in the non-diabetic retinopathy control eyes ${ }^{4}$.

Increase in vascular permeability because of diabetes mellitus brings about extravasation of plasma constituents into the retina prompting DME; Vascular endothelial growth factor (VEGF) is documented to be basic underlying pathogenic factor for development and progression of macular edema auxiliary to Diabetes $^{5}$. VEGF is produced by normal retinal pigment epithelial cells under hypoxic stress. Eyes with substantial spillage from retinal microvasculature leading to thickening of macula have altogether higher VEGF levels when contrasted with eyes with less spillage. Subsequently, anti VEGF agents are reflected as an adjunctive treatment for DME5 ${ }^{5}$. Anti VEGF therapy can lead to decreased leakage from blood vessels $^{6}$

Bevacizumab is a full-length monoclonal humanized antibody that binds and blocks all isoforms of the VEGF-A family. Food and drug administration (FDA) has approved its use in the management of colorectal carcinoma ${ }^{5}$. Currently its use in ophthalmology is off-label. Numerous studies have suggested the prophylactic use of either anti VEGF or steroids as an intravitreal injection after phacoemulsification in patients with diabetic exudative maculopathy to improve the final surgical outcomes in terms of visual acuity and to keep the increase in thickness of macula ${ }^{3}$.

The objective of the study was to determine frequency of macular edema in patients of mild to moderate non-proliferative diabetic retinopathy (NPDR) receiving peroperative intravitreal bevacizumab, as compared to controls, at completion of 1 month after phacoemulsification cataract surgery.

To assess the efficacy of bevacizumab given through intravitreal injection at the conclusion of standard surgery for cataract extraction on the development of diabetic retinopathy (DR) and diabetic maculopathy postoperatively. Participants were randomized to an institutionalized strategy of cataract surgery along with intraocular monofocal lens implantation alone (control group; 30 eyes) or to get an intravitreal injection of $1.25 \mathrm{mgbevacizumab}$ towards the finish of cataract extraction procedure (IVB group; 27 eyes). Visual acuity with and without correction, optical coherence tomography (OCT), and dilated fundoscopic examination were the viablility measures checked monthly postoperatively for a total 6-months follow-up period. There was no noteworthy contrast in central macular thickness (CMT), BCVA, or foundational condition between the control and IVB bunches at gauge. Progression of diabetic retinopathy was documented in 15 among 30 eyes (50\%) in the control group and 2 among 27 eyes $(7.4 \%)$ in the intervention group $(\mathrm{P}=0.0008) .1 .25 \mathrm{mg}$ bevacizumab injection given through intravitreal route on conclusion of cataract surgery was established to be efficient in keeping the progression of DR and diabetic maculopathy in patients with DR undergoing cataract surgery ${ }^{7}$. This study is used as reference study to calculate sample size.

A study published in Korean journal of ophthalmology in 2011 showed that 18\% of the diabetic patients undergoing cataract develop macular edema, with the highest incidence at the conclusion of 1 month after cataract surgery ${ }^{8}$, therefore outcome was measured at 1 month post-operatively.

Rationale of our study was to determine effectiveness of preoperative intravitreal bevacizumab in prophylaxis of macular edema in patients with mild to moderate diabetic retinopathy undergoing phacoemulsification so as to improve the visual outcomes. This study has not been previously done in Pakistani population.

\section{MATERIAL AND METHODS}

A Randomized control trial study was carried out to highlight that the peroperative intravitreal bevacizumab is effective in prophylaxis of macular edema in patients with mild to moderate NPDR, when compared to controls. Study population included patients who reported at hospital with cataract and diabetic retinopathy. Sample size for the study was taken by non-probability, consecutive sampling form a total of 60 participants divided into two groups, The allocation was done through simple random sampling technique by formulating a list of randomly allocated 60 numbers in random number list generated through SPSS, 30 to group A or study group and 30 to control group (B).The study was carried out for a period of 6 months (20th July 2016 to $19^{\text {th }}$ January 2017). 
For the procedure all participants were randomly allocated in a 1:1 ratio to either have an injection of bevacizumab $(1.25 \mathrm{mg}$ in $0.05 \mathrm{ml})$ through intravitreal route on the conclusion of surgery (study group) or not (control group). A thorough ocular examination and OCT was done 1 week prior to the surgery and then 1 month post surgery by the same researcher in all cases. The researcher was not aware of the allocation of the patients to the study groups. The standard ocular examination at each visit comprised of slit lamp examination, uncorrected and best corrected visual acuity, and quantification of the central macular thickness with OCT as an average of three consecutive measurements. Patients underwent standard cataract surgery (phacoemulsification and monofocal intraocular lens (IOL) implantation). One surgeon performed all the surgeries and intravitreal injections. Postoperatively all patients were prescribed moxifloxacin-dexamethasone eye drops two hourly for two weeks followed by tapering over 1 month. Every patient was followed-up and was reassessed at completion of 1 month after baseline and occurrence of macular edema based on central macular thickness was the outcome variable.

Results of the collected data were entered into SPSS version 22.0. Numerical variables were described as mean and standard deviation. While qualitative variables that included the macular edema, the diabetic maculopathy, central macular thickness and side of eye were reported in the form of frequency and percentages.

\section{RESULTS}

Sample was calculated from a total of 60 patients. Mean age of the study participants was reported as $61.97 \pm 5.70$ years. Out of the 60 participants $33(55.0 \%)$ were females while female to male ratio was 1.2:1. Macular edema was seen in $04(13.33 \%)$ patients in study group and $17(56.67 \%)$ patients in control group. Sample selected showed that $02(12.50 \%)$ patients reported with right eye macular and 02 (14.29\%) noticed with left eye in group A while in group B for the right eye macular edema was reported in 08 $(53.33 \%)$ of patients and $09(60.0 \%)$ reported with left eye.

The Gender wise sample collection of patients is described in the pie chart given below (Fig. 1).

Fig. 1: Gender wise distribution of the patients.

The Central Macular thickness in group A was $233.23 \pm 30.16 \mu \mathrm{m}$ and in group B was $253.21 \pm 22.34$ $\mu \mathrm{m}$. Macular edema was seen in $04(13.33 \%)$ patients in study group and $17(56.67 \%)$ patients in control group ( $\mathrm{p}$-value $=0.0001)$. Distribution of patients according to side of eye is shown in figure 2 below.

Fig. 2: Distribution of patients according to side of eye.

Stratification of macular edema with respect to age groups showed significant difference in macular edema in all age groups among both groups. Similarly, statistically significant difference was found in macular edema in female among both groups. Stratification of macular edema with respect to side of eye has shown in Table 1 below.

Table 1: Stratification of macular edema according to side of eye.

\begin{tabular}{|c|c|c|c|c|c|}
\hline \multirow[t]{2}{*}{ Side of eye } & \multicolumn{2}{|c|}{$\begin{array}{l}\text { Group A }(n=30) \\
\text { Macular Edema }\end{array}$} & \multicolumn{2}{|c|}{$\begin{array}{l}\text { Group B }(n=30) \\
\text { Macular Edema }\end{array}$} & \multirow[t]{2}{*}{ P-value } \\
\hline & Yes & No & Yes & No & \\
\hline Left & $02(14.29 \%)$ & 12 (85.71\%) & $09(60.0 \%)$ & $06(40.0 \%)$ & 0.0001 \\
\hline Right & $02(12.50 \%)$ & $14(87.50 \%)$ & 08 (53.33\%) & $07(46.67 \%)$ & 0.015 \\
\hline
\end{tabular}

As the details of Macular edema found in both groups are graphically shown in figure 3 below.

Fig. 3: Distribution of patients according to macular edema in both Groups.

\section{DISCUSSION}

Diabetic maculopathy is a foremost cause of loss of visual acuity in diabetic population ${ }^{9}$. It is well-known fact that outcomes of cataract surgery in terms of visual acuity can be negatively affected by DME. ${ }^{10} \mathrm{It}$ was suggested by some authors that any clear evidence is lacking to show that phacoemulsification cataract surgery causes advancement of DME, specifically in low-risk population; i-e, those with mild diabetic retinopathy or with controlled retinal disease. However, it was established by Kim et $\mathrm{al}^{11}$ that $22 \%$ of the diabetic patients undergoing uncomplicated phacoemulsification show $>30 \%$ increase in central retinal thickness as quantified by optical coherence 
tomography (OCT). Numerous studies have proposed the prophylactic use of intravitreal injection of either bevacizumab or steroids in patients with DME that are undergoing phacoemulsification to improve the final anatomical and physiological outcome of intervention in terms of retinal thickness and visual acuity. ${ }^{12-15}$ I have conducted this study to determine frequency of macular edema in patients of mild to moderate nonproliferative diabetic retinopathy (NPDR) receiving peroperative Intravitreal bevacizumab, as compared to controls, at completion of 1 month after phacoemulsification cataract surgery.

Age range in this study was from 50 to 70 years with mean age of $61.97 \pm 5.70$ years. The participants in group A (study group) had a mean age of $61.60 \pm$ 5.75 years while that of participants in group B was $62.33 \pm 5.71$ years. Majority of the patients $38(63.33 \%)$ were between 61 to 70 years of age. Out of 60 patients $33(55.0 \%)$ were females and $27(45.0 \%)$ were males with female to male ratio of 1.2:1. Macular edema was seen in $04(13.33 \%)$ patients in study group and17 $(56.67 \%)$ patients in control group ( $p$-value $=0.0001)$. The purpose of this study was to assess adequacy of bevacizumab given as an intravitreal injection at the conclusion of cataract surgery on the postoperative outcomes of surgery in terms of diabetic retinal changes. For this purpose all the participants were randomly allocated in a 1:1 ratio to either have an intravitreal injection of bevacizumab $(1.25 \mathrm{mg}$ in $0.05 \mathrm{ml}$ ) at the completion of surgery (study group) or not (control group). Visual acuity with and without correction, optical coherence tomography (OCT), and dilated fundoscopic examination were the efficacy parameters checked monthly for a 6 months postintervention follow-up period. Noteworthy contrast was not seen in central macular thickness (CMT), BCVA, or foundational condition between the control and IVB groups at baseline. Progresson of diabetic retinopathy was documented in 15 among 30 eyes $(50 \%)$ in the control group and 2 among 27 eyes $(7.4 \%)$ in the intervention group $(\mathrm{P}=0.0008)$.

In a study ${ }^{16}, 42$ patients with diabetic macular edema were randomly allocated to either phacoemulsification only or combined with intravitreal injection of bevacizumab. Macular thickness (MT) as quantified by optical coherence tomography was $\square 300 \mu \mathrm{m}$ in all patients. The eyes with PDR or those that had received laser treatment at least 1 year before surgery were excluded. Reassessment of CMT at first and third month after surgery, demonstrated a substantial decrease in the intravitreal bevacizumab group in comparison to no bevacizumab group in which it increased significantly. Likewise bevacizumab group showed significantly higher improvement in VA as compared to the control group; which was correlated to be due to the reduction in MT in the bevacizumab group ${ }^{16}$.

Cheema and colleagues ${ }^{17}$ investigated as to whether intravitreal bevacizumab injection with cataract surgery prevents postoperative diabetic macular edema (PME) in patients with stable DR with no notable ME. Eighty patients each with visually significant lenticular opacity, steady preproliferative DR, and no substantial ME were randomized to a sham group (cataract surgery only), or a study group undergoing cataract surgery with bevacizumab injection at the end of procedure. Best-corrected visual acuities, central subfield foveal thickness, and macular volume were measured by means of optical coherence tomography at baseline and then 1 week, 1, 3, and 6 months postoperatively. Clinically significant postoperative macular edema (PME) was defined as $>60 \mu \mathrm{m}$ increase in central subfield thickness as compared to baseline. There was no significant difference in measured parameters at baseline. The sham group showed significantly larger increment in central subfield thickness at 1 week and 1 month postoperatively as compared to baseline, larger increases in total macular volume at all follow ups, greater development of PME at 1 month follow up, and poorer best-corrected visual acuities outcome from baseline to 6 months post intervention. It was concluded from the study that, intravitreal injection of bevacizumab given at the time of surgery in patients with stable DR without significant ME, undergoing cataract surgery might be effective in preventing the postoperative worsening of $\mathrm{ME}$ and it might improve the concluding visual outcome of cataract surgery ${ }^{17}$.

In another study ${ }^{18}$, participants were randomly allocated to a control group undergoing standard procedure of cataract extraction followed by intraocular lens implantation alone or intervention group to get an intravitreal injection of standard dose of bevacizumab on the conclusion of cataract surgery. Patients were monitored postoperatively up to a duration of 6 months specifically for development and progression of any retinal and macular changes attributed to diabetes. In total sixty-eight eyes were enrolled in the study. Diabetic retinal changes progressed in $15(45.45 \%)$ among 33 eyes in the cataract surgery only group and $4(11.42 \%)$ among 35 eyes in the cataract surgery plus bevacizumab group 
$(\mathrm{P}=0.002) .17$ eyes $(51.51 \%)$ in the control group showed progression of maculopathy as compared to only 2 eyes $(5.71 \%)$ in the intervention group $(\mathrm{P}=$ 0.0001). But visual acuities did not show any significant contrast between the 2 groups postoperatively $(\mathrm{P}=0.772)$; however, two eyes in the control group progressed to neovascular glaucoma as compared to none in intervention group. No significant difference was seen between the mean postoperative central macular thickness and mean macular thickness between the 2 groups $(\mathrm{P}=0.874$ and 0.942 , respectively) ${ }^{18}$.

An interventional, randomized, open-label and control study ${ }^{19}$ of two parallel groups of already diagnosed patients with pre-proliferative diabetic retinopathy without macular edema was done. The study included sixty eyes of sixty patients, having non-proliferative diabetic retinopathy without macular edema and lens opacity (grade 1 to 3). One group $(\mathrm{n}=30)$ received intra-vitreal injection of Bevacizumab and the control group $(n=30)$ did not received intra-vitreal injection of Bevacizumab during standard phacoemulsification. Best-corrected visual acuity (BCVA) on the conclusion of two months compared with the baseline visual acuity recorded along with central macular thickness (CMT) measured on optical coherence tomography (OCT) was the key outcome measured. The mean age of the patients was also similar in the control (55.2 \pm 9.66 years) and Bevacizumab groups ( $56.47 \pm 9.13$ years) ranging from 40 to 75 years. All of the patients in Bevacizumab group had visual acuity of 6/6postoperatively except one eye $(3.33 \%)$ had $6 / 12$ due to CSME that was evident on OCT as increase in macular thickness. While in control group 10 (33.33\%) eyes out of 30 had BCVA of $6 / 12$ or less (evident on OCT as increase in macular thickness $)^{19}$.

Another study carried out by Lanzagorta et al ${ }^{12}$ has shown improvement in the vision and decrease in the retinal thickness in the Bevacizumab group in contrast to control group. Mason et $\mathrm{al}^{20}$ described noticeable improvement of visual acuity in 2 patients with persistent CME which has been effectively treated with Bevacizumab. On the whole, it was concluded that peroperative intravitreal bevacizumab is effective in prophylaxis of macular edema in patients with mild to moderate NPDR, as compared to controls, at completion of 1 month after phacoemulsification cataract surgery.

\section{CONCLUSION}

The study concludes that the peroperative intravitreal bevacizumab is effective in prophylaxis of macular edema in patients with mild to moderate NPDR, as compared to controls, at completion of 1 month after phacoemulsification cataract surgery. So, we recommend that peroperative intravitreal bevacizumab should be used as a prophylaxis of macular edema in patients with mild to moderate NPDR after phacoemulsification cataract surgery.

\section{Author's Affiliation}

Dr. Sidra Jabeen

F CPS, Ohthalmology department

Holy family hospital, Rawalpindi

Dr. Rizwan Khan

FCPS, Senior registrar, Ophthalmology department Holy family hospital, Rawalpindi

Dr. Ali Raza

MCPS, FCPS, Professor, ophthalmology department

Holy family hospital

\section{Role of Authors}

Dr. Sidra Jabeen

Corresponding author, Planning of research including data collection, collection methods, setting, collection of data, drafting.

Dr. Rizwan Khan

All surgical interventions done, analaysis and interpretation of data.

Dr. Ali Raza

Critical revision of all work, supervisor of research project.

\section{REFERENCES}

1. Qazi HA. Intravitreal administration of $1.25 \mathrm{mg}$ bevacizumab at the time of cataract surgery was safe and effective in preventing the progression of DR and diabetic maculopathy in patients with cataract and DR. J Res Med Sci. 2012; 17: 1180-1187.

2. Chae JB, Joe SG, Yang SJ, Lee JY, Sung KR, Kim JY, Kim JG, Yoon YH. Effect of combined cataract surgery and ranibizumab injection in postoperative macular edema in nonproliferative diabetic retinopathy. Retina, 2014; 34: 149-56.

3. Brito PN, Rosas VM, Coentrão LM, Carneiro ÂV, Rocha-Sousa A, Brandão E, Falcão-Reis F, Falcão MA. Evaluation of visual acuity, macular status, and subfoveal choroidal thickness changes after cataract 
surgery in eyes with diabetic retinopathy. Retina, 2015; 35: 294-302.

4. Ateeq A, Tahir MA, Cheema A, Dahri A, Tareen S. Intravitreal injection of Bevacizumab in diabetic macular edema. Pak J Med Sci. 2014; 30: 1383-7.

5. Salehi A, Beni AN, Razmjoo H, Beni ZN. Phacoemulcification with intravitreal bevacizumab injection in patients with cataract and coexisting diabetic retinopathy: prospective randomized study. J Ocul Pharmacol Ther. 2012; 28: 212-8.

6. Bonnin S, Dupas B, Lavia C, Erginay A, Dhundass M, Couturier A, Gaudric A, Tadayoni R. Antivascular endothelial growth factor therapy can improve diabetic retinopathy score without changes in retinal perfusion. Retina. 2019 Mar; 39(3): 426-434.

7. Kwon SI, Hwang DJ, Seo JY, Park IW. Evaluation of changes of macular thickness in diabetic retinopathy after cataract surgery. Korean J Ophthalmol. 2011; 25 (4): 238-42.

8. Moss SE, Klein R, Klein BEK. The incidence of vision loss in a diabetic population. Ophthalmol. 1998; 95 (10): 1340-1348.

9. Nelson L, Martidis A. Managing cystoid macular edema after cataract surgery. Curr Opin Ophthalmol. 2003; 14 (1): 39-43.

10. Shah AA, Chen SH. Cataract surgery and diabetes. Curr Opin Ophthalmol. 2010; 21 (1): 4-9.

11. Kim SJ, Equi R, Bressler NM. Analysis of macular edema after cataract surgery in patients with diabetes using optical coherence tomography. Ophthalmol. 2007; 114 (5): 881-889.

12. Lanzagorta-Aresti A, Palacios-Pozo E, Menezo Rozalen JL, Navea-Tejerina A. Prevention of vision loss after cataract surgery in diabetic macular edema with intravitreal bevacizumab: a pilot study. Retina, 2009; 29
(4): 530-535.

13. Takamura Y, Kubo E, Akagi Y. Analysis of the effect of intravitreal bevacizumab injection on diabetic macular edema after cataract surgery. Ophthalmol. 2009; 116 (6): 1151-1157.

14. Akinci A, Muftuoglu O, Altınsoy A, Ozkılıc E. Phacoemulsification with intravitreal bevacizumab and triamcinolone acetonide injection in diabetic patients with clinically significant macular edema and cataract. Retina, 2010; 31 (4): 755-758.

15. Elman MJ, Aiello LP, Beck RW, Bressler NM, Bressler SB, Edwards AR, et al. Randomized trial evaluating ranibizumab plus prompt or deferred laser or triamcinolone plus prompt laser for diabetic macular edema. Ophthalmology, 2010 Jun. 117 (6): 1064-1077.e35.

16. Ahmed M, Nawaz M, Javed EA, Sultan M. Efficacy of Intra-Vitreal Bevacizumab combined with PhacoEmulsification in the Prophylaxis of Macular Edema in Patients with Non-Proliferative Diabetic Retinopathy. APMC. 2016; 10 (2): 58-62.

17. Cheema RA, Al-Mubarak MM, Amin YM, Cheema MA. Role of combined cataract surgery and intravitreal bevacizumab injection in preventing progression of diabetic retinopathy: Prospective randomized study. J Cataract Refract Surg. 2009; 35: 18-25.

18. Klein R. The Diabetes Control and Complications Trial. Kertes C, ed. Clinical Trials in Ophthalmology: A Summary and Practice Guide, 1998: 49-70.

19. Akduman L, Olk RJ. The early treatment for diabetic retinopathy study. Kertes C, ed. Clinical Trials in Ophthalmology: A Summary and Practice Guide, 1998: 15-36.

20. Mason JO, Albert MA, Vail R. Intravitreal bevacizumab (Avastin) for refractory pseudophakic cystoid macular edema. Retina, 2006; 26: 356-7. 